\title{
The Secondary Optical Design and Fabrication for the Uniform Illuminating LED Spotlight Using TIR Lens
}

\author{
Yingchao Xu ${ }^{a, 1,2}$, Guanyu Chen ${ }^{1,2}$, Yongxiao Chang ${ }^{1,2}$, Hongyi Lin ${ }^{1,2}$, Jiamei Zheng ${ }^{1,2}$, Liangqin Gan ${ }^{1,2}$ \\ ${ }^{1}$ School of Opto-electronics and Communication Engineering, Xiamen University of Technology, Xiamen 361024, China \\ ${ }^{2}$ Fujian Provincial Key Laboratory of optoelectronic information materials and devices, Xiamen University of Technology, Xiamen \\ 361024, China
}

\begin{abstract}
Different with the fact that the traditional imaging optics emphasizes its ability to get clear image on the image plane, the purpose of LED illumination design is to meet the requirement of high availability of optical energy and homogeneity of illumination. As the study object, the LED track lighting has been designed with secondary optical design by the Lighttools design software. After using TIR lens which has two ways of concentrating: reflectors and lenses, the exit light of the spotlight will be adjusted and the illuminated surface will be with high homogeneity. Therefore, it will reduce the harm of the light to human's eyes. In this study, the LED spotlights have been produced and tested with the corresponding test results: Lighting power is $7 \mathrm{~W}$, luminous efficiency is $82.57 \mathrm{~lm} / \mathrm{W}$, color rendering index is 83.7 , the main wavelength is $488.2 \mathrm{~nm}$, the ratio of red, green, blue is 13.1: 83.0: 3.9 .
\end{abstract}

\section{Introduction}

The implementation of the "Ten cities, ten thousand lights" project in China and the LED lighting demonstration project for Beijing Olympics and Shanghai World Expo greatly enhanced the development speed of the LED lighting. Known as the fourth generation lighting source, LED's development is very rapid. And it is a trend to replace the traditional energysaving lamp light sources gradually[1-2]. As the luminous efficiency of LED increases, its advantages such as the full color, environmental protection, energy saving become more and more significant. Although LED has already entered the field of lighting, the problem of secondary light distribution restricts its further development. LED's application will shine in the field of lighting, when the bottlenecks of secondary light distribution[3-4] and heat-release are solved. The development of advanced lighting design software and the appearance of free surface optical components, making the research works of improving secondary light distribution and the efficiency of LED lighting are more and more convenient.

In recent years, the research works have done on the secondary light distribution design of the LED sources at home and abroad. For example, LED array illumination light source and surface light source[3-4], the research on LED lamp[5], the secondary light distribution design on LED spotlights are also reported in the literature[6] , by adjusting each individual Fresnel lens ring with the inclination to optimize the distribution of the projected light. So far greater advantage of the Fresnel lens is applied in terms of the focus of light in the secondary light distribution field, but it is not the best means.

In this paper, we use the LED track spotlight as the example. Using the TIR lens as the light-uniform element has advantages that it integrates the reflector and the converging lens. Ultimately uniform illumination is achieved at the irradiated surface. We manufactured the LED lamp and the photoelectric parameter test results were given in the paper.

\section{Introduction of TIR Lens And Its}

\section{Performance Evaluation}

TIR lens is the total internal reflection lens, using the principle of total reflection optics, to collecte and process light. Fig.1 Shows the sketch of the TIR lens. TIR lens can complete the collection of light, and we can achieve a beam angle control by adjusting the radius of curvature of the TIR lens.

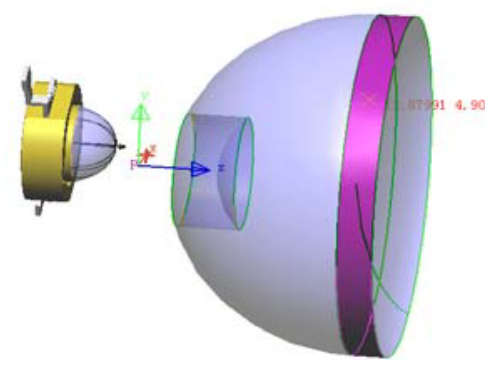

Figure 1. The sketch of the TIR lens

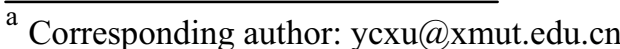




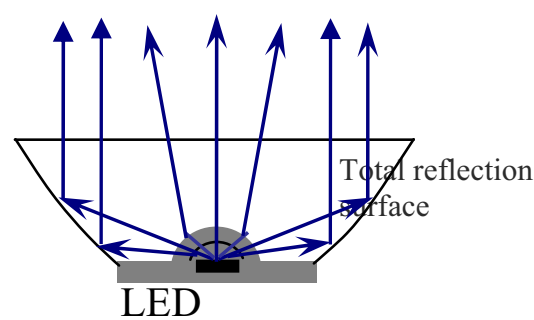

Figure 2. The diagram of the light distribution effect for TIR lens

Lens has the excellent light-gathering ability for the rays at a small angle. While the reflector has the advantage to converging the rays at a large angle. So both the lens and the reflector are combined together, as shown in Figure 2. Reflector and lens group combination into TIR lens, can effectively improve the luminous efficiency of LED lighting devices, avoid the waste of LED luminescence. In summary, Using the combination of the reflector and the lens, addition to the use of imitation compound eye micro lens array, the light concentrating ability of the LED lamp will be greatly improved. The efficiency and the uniformity will be improved greatly ${ }^{[7]}$. By adding $3 \mathrm{D}$ texture, we can also eliminate the glare of the lamp and the eye discomfort.

In beam shaping process of the LED light source, although tapered light pipe (TLP) [8] and compound parabolic concentrator (CPC)[9] both have very high optical collectting efficiency, but in order to improve the intensity of illumination uniformity of the system, usually makes the system very long. Obviously, in improving the illumination uniformity and reducing the size of the system to make the system more compact simultaneously, TIR lens has more advantages. So we adopt the TIR lens as secondary light distribution element for LED track lamp.

For the performance evaluation of TIR lens used in the illumination system, we mainly use the light energy utilization ratio and intensity of illumination uniformity [10].

\section{[1]. Light energy utilization ratio}

By calculating the ratios of luminous flux that reaches the target screen receiver and the total light flux emitted by LED light source, we can get the energy utilization of the system. The computation formula is as follows:

$$
\eta^{\prime}=\phi_{\text {screen }} / \phi_{L E D}
$$

\section{[2]. Intensity of illumination uniformity}

By dividing the target screen into $m \times n$ grid, and through the calculation of intensity of illumination $B$ of each box ,we can work out the error between each region and the mean value $\mathrm{S}$ :

$$
S=\sqrt{\frac{1}{m \times n-1} \sum_{i=1}^{m \times n}\left(B_{i}-\bar{B}\right)^{2}}
$$

Calculated error $\mathrm{S}$, and based on the average $\bar{B}$ has been calculated, seeking both ratios, you can get illuminance uniformity:

$$
U=\frac{S}{\bar{B}}
$$

In the fomula above, the smaller of the error $\mathrm{S}$, the smaller of the intensity of illumination uniformity. That the intensity of illumination uniformity is the better.

\section{Lighting System Design}

In order to eliminate the harm to the human's eyes caused by the glare of the LED lamp, lighting system design must be executed. And the TIR lens is used to obtain better illumination uniformity

The light source used is the patch type white LED Lambertian light source, with a divergence angle of $120^{\circ}$. The radiation intensity has nothing to do with the viewing angle, the radial brightness on the various angles are the same. Therefore, the light scattering characteristics as follows: the scattering surface is arbitrary; the probability of each scattering direction is same; Scattering follow normal direction rather than on the surface of the incidence angle; Each of the scattering light have the same energy. Light intensity emitted from the light source into a cosine distribution, formation of the spot center is very bright, along the radial attenuation [11].

Through research, we found that the arrangement have less effect on the intensity of illumination uniformity and light energy utilization rate, but the distance between LEDs on both greatly influenced. For rectangular arrangement of six LED module systems, the utilization of light energy decreases gradually with the increasing of the distance between the LED lights, and illumination uniformity decreases with the increase of distance, and then decreases with the increasing of the distance. For the annular arrangement,light energy utilization ratio decreases with increasing distance, intensity of illumination uniformity decreases with increasing distance change trend [12].

In this design, in accordance with the lamp bead emission angle with the scattering angle and uniformity of lens and light energy utilization and the relationship between the distance, the arrangement of seven LED lamp bead every three forms an equilateral triangle, the angle is $60^{\circ}$ and distance is $2.2 \mathrm{r}$ ( $\mathrm{r}$ is the radius of the lens). The arrangement is shown in Figure 3, each dot represents a single LED lamp bead. 


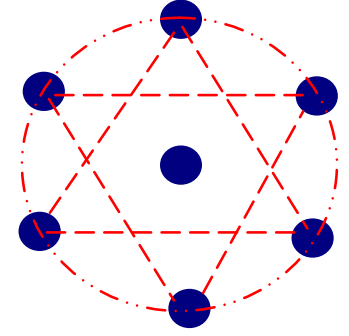

Figure 3. The arrangement of the seven LED lamps

According to the theory of nonimaging optics, the purpose of secondary light distribution design is to change the LED light Angle and optical intensity distribution, in order to satisfy the demands of the corresponding lighting.

Light distribution curve according to the beam angle is usually divided into:

Narrow light distribution $\left(<20^{\circ}\right)$

Medium light distribution $\left(20^{\circ}-40^{\circ}\right)$

Wide light distribution $\quad\left(>40^{\circ}\right)$

Because TIR lens is essentially a lens at center area, mainly for the light at small angles to carry out light distribution design. The surrounding area of the TIR lens is total reflection, mainly for the light at the large angles to carry out the secondary light distribution design, so TIR lens deals with the most perfect illumination uniformity.

In the design we use Lighttools lighting design software.Total reflection of both the middle section of lens, and peripheral reflection section, are used with quadric surfaces (it could be a parabolic, ellipsoid or hyperboloid). In fact which we used are hyperboloid surface shape. So that each surface is more than the traditional spherical surface of a quadric coefficient variation. In actual optimal design process, we set the two quadric coefficients of the surfaces for the lens in the center and peripheral total reflector as two optimization variables. And then add collimating optimization function, run ray tracing. The collimation embedded in the Lighttools software design optimization function, its principle is to use Monte Carlo ray tracing method will all fall in the cosine of the light receiving surface consistent.Final design layout and illumination intensity distribution is shown in figure 4 . According to the following design results and formula (2), (3), we can get error $\mathrm{S}=74.81, \mathrm{U}=33.85 \%$.

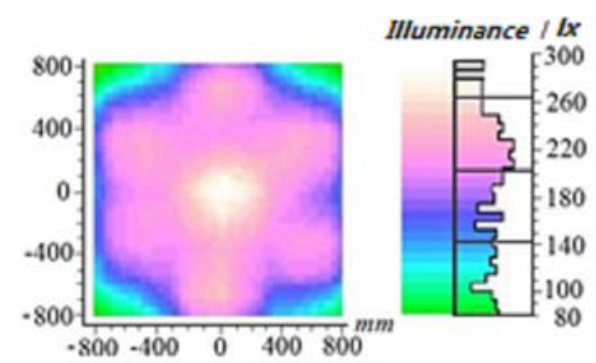

(b)

\begin{tabular}{|l|l|l|l|}
\hline 101 & 192 & 195 & 109 \\
\hline 274 & 299 & 292 & 279 \\
\hline 281 & 298 & 296 & 280 \\
\hline 118 & 198 & 189 & 135 \\
\hline
\end{tabular}

(c)

Figure 4. The illumination intensity effect of the lamp after optimized

(a) The diagram of the ray tracing for the lamp (b) The illumination intensity $(c)$ The value of the illumination intensity divided by the grid of $16 \times 16$

The micro lens array stage imitating compound eyes is also used in lighting system,Its aim straight design for lighting uniformity has a good effect[13]。Therefore we have to add the above design a good set of TIR lens design of micro lens array (As shown in figure 5 ). According to the design results and formula shown below (2), (3), we are able to get the error $\mathrm{S}=40.43, \mathrm{U}=$ $19.18 \%$. Design result of light intensity of illumination figure shows that the illumination uniformity is improved significantly. But the disadvantage is that due to the addition of a micro lens array, the scattering light to the periphery has a small part of the increase. The average illumination of the lamp is reduced to $210.751 \mathrm{x}$ by $2211 \mathrm{x}$, reduce the rate of $4.64 \%$. In the main pursuit of the effect of the illumination uniformity, reducing the harm of the lamp glare on the human eye, the light energy utilization rate of the range is acceptable.

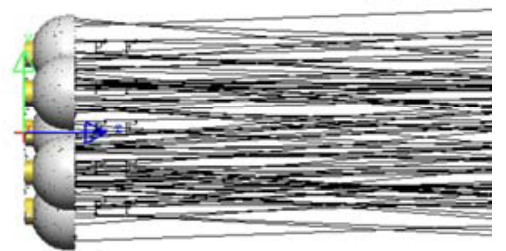

(a)

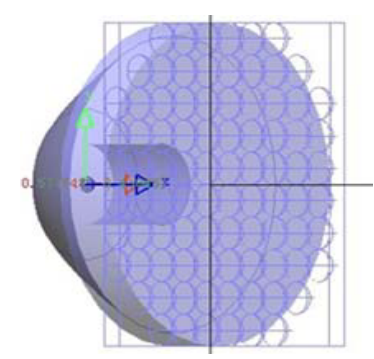

(a) 


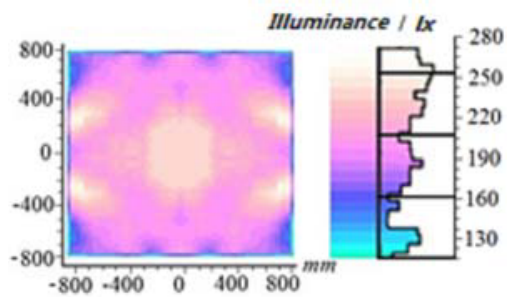

(b)

\begin{tabular}{|l|l|l|l|}
\hline 155 & 208 & 211 & 159 \\
\hline 236 & 264 & 248 & 228 \\
\hline 234 & 266 & 247 & 231 \\
\hline 150 & 190 & 197 & 148 \\
\hline
\end{tabular}

(c)

Figure 5. The micro-lens array for the TIR lens and the improvement of the illumination intensity uniformity (a) The micro-lens array added for the TIR lens (b) The illumination intensity (c) The value of the illumination intensity divided by the grid of $16 \times 16$

\section{The Manufacture Of Lamps And Testing}

\subsection{The manufacture of the lamp}

According to the driving characteristics of LED lamp, we designed the corresponding driver circuit, as shown in figure 6. Including voltage transformer, rectifier, filter, voltage regulator and other circuits.etc.

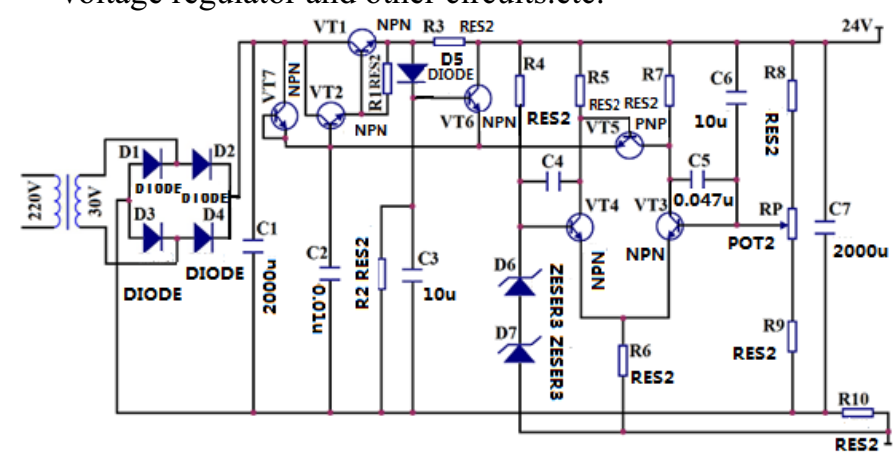

Figure 6. Driving circuit for LED light

We used Solidworks software to design the lamp housing, cooling systems, metal ring groove that fixed TIR lens. Ultimately,we obtained the the corresponding design and the manufactured LED track lamp is shown in Figure 7.

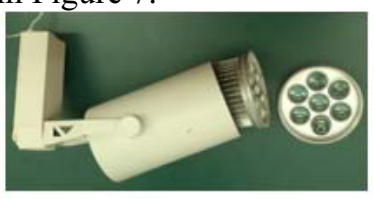

(a)

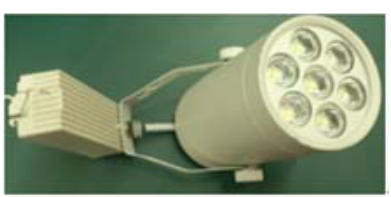

(b)
Figure 7. The picture of the LED track spotlight

\subsection{Lamp Test}

The light intensity distribution curve of the lamp was tested by GO2000A which produced by Hangzhou Everfine Ltd.. The light intensity distribution curve is shown in Figure 8 and the basic parameters of the light source as shown in Table 1.

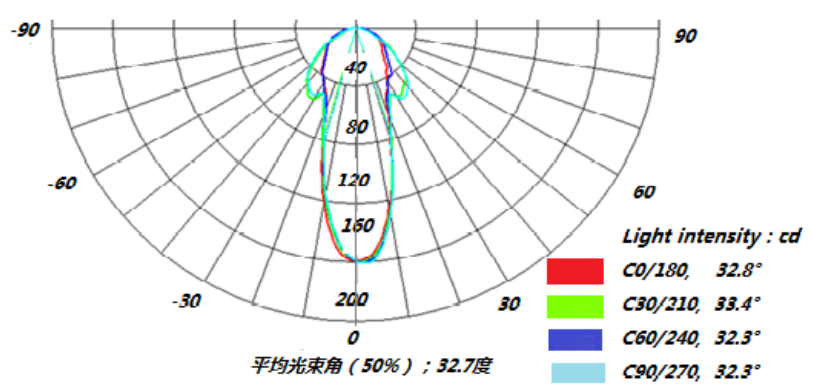

Figure 8. The curve of the intensity distribution

From Figure 8, we can see that the lamp light distribution is within \pm 15 degrees, the average beam angle is 32.7 degrees, belonging to medium light distribution. The photoelectric parameters of the lamps were measured by integrating sphere, as shown in Figure 9 and figure 10. The results of the measurements are shown in the following table 1.

Table 1. Test results fixtures

\begin{tabular}{|l|l|l|l|l|l|l|}
\hline Total power & $7 \mathrm{~W}$ & \multicolumn{2}{|l|}{ Luminous efficiency } & \multicolumn{2}{l|}{$82.57 \mathrm{~m} / \mathrm{W}$} \\
\hline Peak intensity & $\begin{array}{l}16306 \\
\mathrm{~cd}\end{array}$ & \multicolumn{2}{|l|}{ The total flux } & $577.991 \mathrm{~m}$ \\
\hline $\begin{array}{l}\text { Average beam } \\
\text { angle }\end{array}$ & $32.7^{\circ}$ & \multicolumn{2}{|l|}{ Color Temperature } & $6270 \mathrm{~K}$ \\
\hline $\begin{array}{l}\text { Dominant } \\
\text { wavelength }\end{array}$ & $\begin{array}{l}488.2 \\
\mathrm{~nm}\end{array}$ & \multicolumn{2}{|c|}{ Peak wavelength } & \multicolumn{2}{|l|}{$450.0 \mathrm{~nm}$} \\
\hline $\begin{array}{c}\text { Red } \\
\text { ratio } \\
(\%)\end{array}$ & $\begin{array}{c}\text { Green } \\
\text { ratio } \\
(\%)\end{array}$ & 83.0 & $\begin{array}{c}\text { Blue } \\
\text { ratio } \\
(\%)\end{array}$ & 3.9 & $\mathrm{Ra}$ & 83.7 \\
\hline
\end{tabular}

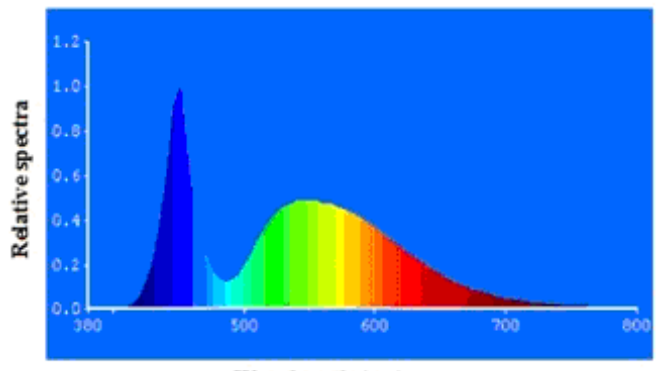

Wavelength $(\mathrm{nm})$

Figure 9. The spectral curve of the lamp 


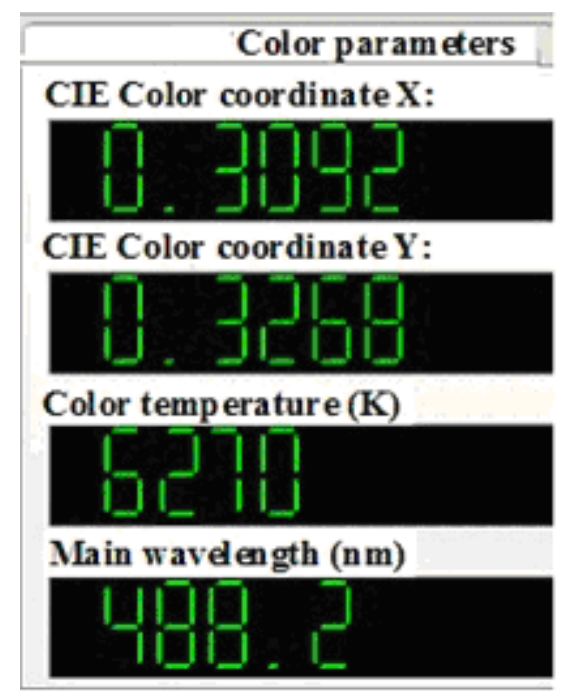

Figure 10. The photometric test reslut of the LED lamp

After testing, we obtain that the power of the LED lamp is $7 \mathrm{~W}$, the luminous efficiency is $82.57 \mathrm{~lm} / \mathrm{W}$, the color rendering index is 83.7 . From Figure 10 we can see the main wavelength is $488.2 \mathrm{~nm}$, and the ratio of red light, green light, and blue light is 13.1: 83.0: 3.9.

\section{Conclusion}

For LED lamps which just has been carry out the first light distribution, the light is not all projected to the expected range, but also after the package is not uniform distribution of light intensity, the illumination area always appears to diffuse. The secondary light distribution can make up for the waste of energy and distribution of unequal defects, and it can effectively eliminate glare, improve LED lighting illumination uniformity.

From the results of the design, manufacture and testing, the technical parameters of the LED track lamp in this paper has reached a good level in all aspects. In many indicators, especially for the process of illumination uniformity achieved superior market LED track lamp levels, it can be widely used clothing stores or large stage lighting and other aspects of the configuration, and highly practical.The development of the secondary light distribution in this paper for the new generation of LED lighting has a certain reference value for the same industry .

\section{Acknowledgment}

This work is supported by the National Natural Science Foundation of China (11304259) and the S\&T plan projects of Fujian Provincial Education Department (JA12254)

\section{References}

1. R.lenk,C.Lenk,Practical lighting design with LEDs(IEEE Press, Wiley,2011)

2. R. Winston, J.C. Miflano, P. Benitez. Nonimaging Optics(Elserier, Amsterdam, 2005)

3. C.Sommer,E.Hartmann,P.Pachler.Optical Materials,9 (2008)

4. Chang,Y.-C.,Ou,C.-J.,Juang,F.-S.Opt. Rev.16 (2009)

5. Lee,H.,et al.Opt.Express,,21 (2013)

6. Yi Ding, Peifu Gu, Wei Lu.Engineering Science 41,9 (2007)

7. F.Chen,K.Wang,Z.Qin,D,Wu,X.B.Luo,S.Liu,Opt Express 18,20 (2010)

8. Bol'shukhin, V.A., Ilyasov, V.S., Soshchin, N.P., Ulasyuk, V.N.,J. Opt. Technol 78,7 (2011)

9. C.P.Krijn,A.B.Salters,H.O.Willemsen.SPIE, 6196 (2006)

10. Jiang,J.,To,S.,Lee,W.B,Cheung,B.,Optik,121 (2010)

11. Zhang Kang, Chengyue Su,Qian Fu,Tao Xue,Chunhua Zhang.Journal of Guang Dong University of Technology 30,1 (2013)

12. Wang Bao, Rongbin $\mathrm{Yu}$, Feng-guo Li.China Illuminating Engineering Journal,5 (2013)

13. K.H.Jeong,J.Kim,L.P.Lee.Science,312(2006) 